\title{
Perianesthetic considerations in laparoscopic bariatric surgery
}

\author{
Jin Young Lee, Sung Jin Hong, Ji Young Lee, and Jeong Hee Shin \\ Department of Anesthesiology and Pain Medicine, The Catholic University of Korea College of Medicine, Seoul, Korea
}

Bariatric surgery can be challenging for anesthesiologists because morbidly obese patients have a higher incidence of morbidity and mortality from perioperative cardiopulmonary insufficiency, wound infections, and thromboembolic events. Bariatric surgery is a currently popular treatment for morbid obesity in Korea but the anesthetic guidelines for bariatric surgery may not account for the social and racial differences in the characteristics of obesity and bariatric anesthesia, leading to incorrect estimates of the morbidity and mortality from these procedures. Therefore, we retrospectively reviewed the medical charts of 192 morbidly obese patients who had undergone laparoscopic bariatric surgery at a single hospital and discussed perianesthetic management.

Anesthesia care for the patients undergoing bariatric surgery begins with a pre-anesthetic assessment. Moreover, the existence and severity of co-morbidities, rather than the body mass index (BMI) itself, are the main perioperative risk factors [1]. The overall mortality of bariatric surgery is $<1 \%$ [2]. According to the Obesity Surgery Mortality Risk Score, age 45 years old or older, hypertension, BMI $>50 \mathrm{~kg} / \mathrm{m}^{2}$, male gender and risk of pulmonary embolism have been proposed to predict perioperative mortality [3]. Morbid obesity is associated with decreased lung and chest wall compliance, increased airway resistance, reduced functional residual capacity (FRC), and increased oxygen consumption that predisposes them to rapid desaturation during apnea or hypoventilation [4]. Adequate pre-oxygenation in a head-up position is important before anesthetic induction [5]. This position reduces the pressure from the abdominal contents pushing up against the diaphragm and prolongs the mean desaturation time by approximately $50 \mathrm{~s}$ [6]. Bariatric patients have a high incidence of gastroesophageal reflux and increased intragastric pressure caused by the increased mass of the chest and abdomen, leading to a higher risk of pulmonary aspiration during anesthetic induction [7]. Rapid sequence intubation is necessary using succinylcholine or sufficient dose of rocuronium with the Sellick's maneuver. The Pentax-AWS ${ }^{\circledR}$ and Glidescope ${ }^{\circledR}$ laryngoscope were also useful in patients with potential difficult intubation. Intraoperative fluid management during laparoscopic surgery is complex because the central venous pressure and urine output are unreliable during pneumoperitoneum. Because cold, dry gas is insufflated into the peritoneal cavity, the insensible fluid loss during laparoscopic surgery is comparable to open laparotomy, and large amounts of fluid are needed to prevent postoperative acute tubular necrosis [8]. However, the cardiopulmonary function and fluid tolerance are different in morbidly obese patients. In addition, pneumoperitoneum at $15 \mathrm{mmHg}$ causes significant intraoperative oliguria [9], and decreased renal excretion of excessive fluid during laparoscopic surgery may induce volume overloading. Intraoperative fluid requirements during bariatric surgery are usually reported as $4-5 \mathrm{~L}$ of crystalloid solution for an average $2 \mathrm{~h}$ operation [8]. However, the fluid requirements should be standardized according to the operation time and body weight. We used an average of 6.5 $\mathrm{ml} / \mathrm{kg} / \mathrm{hr}$ of crystalloid solution to replace the insensible loss during surgery and it was seemed appropriate. Morbidly obese patients are more likely to be immobile postoperatively and are at risk of deep vein thrombosis and pulmonary embolism, with

Received: August 18, 2010. Accepted: August 23, 2010.

Corresponding author: Jin Young Lee, M.D., Ph.D., Department of Anesthesiology and Pain Medicine, The Catholic University of Korea College of Medicine, Seoul St. Mary's Hospital, Banpo-dong, Seocho-gu, Seoul 137-701, Korea. Tel: 82-2-2258-6162, Fax: 82-2-537-1951, E-mail: 17035@hanmail.net (c) This is an open-access article distributed under the terms of the Creative Commons Attribution Non-Commercial License (http:// creativecommons.org/licenses/by-nc/3.0/), which permits unrestricted non-commercial use, distribution, and reproduction in any medium, provided the original work is properly cited. 
a combined incidence of approximately $2 \%$ [10]. The prevalence of venous thromboembolism (VTE) shows ethnic variations, and Asians have a 3-5 fold lower incidence of symptomatic first time idiopathic and secondary VTE than other racial groups [11]. Therefore, thromboprophylaxis guidelines should be modified in Korean patients. It is important to consider individual patient characteristics, including races, family history, comorbid conditions, concomitant medications, and perioperative hemostasis. In our patient group, none of the patients had a history of previous thrombotic events. Compression stockings were used routinely for VTE prevention and no thromboembolic events were observed. A further evaluation should be performed to determine the prophylactic management of thrombosis in Koreans.

In conclusion, we report the perianesthetic management in what is to our knowledge the largest series of bariatric surgery in Korea. However, the impact of obesity is significant and should be considered throughout the perioperative period. Overall, anesthesiologists should be aware of the increased risk of difficulties with airway management as well as cardiovascular and respiratory care, and should take appropriate precautions to improve patient safety.

\section{References}

1. Leykin Y, Pellis T, Del Mestro E, Fanti G, Marzano B. Perioperative management of 195 consecutive bariatric patients. Eur J Anaesthesiol 2008; 25: 168-70.

2. Buchwald H, Avidor Y, Braunwald E, Jensen MD, Pories W, Fahrbach $\mathrm{K}$, et al. Bariatric surgery: a systematic review and meta-analysis. JAMA 2004; 292: 1724-37.

3. Kuruba R, Koche LS, Murr MM. Preoperative assessment and perioperative care of patients undergoing bariatric surgery. Med Clin North Am 2007; 91: 339-51.

4. Biring MS, Lewis MI, Liu JT, Mohsenifar Z. Pulmonary physiologic changes of morbid obesity. Am J Med Sci 1999; 318: 293-7.

5. Bagatini A, Trindade RD, Gomes CR, Marcks R. Anesthesia for bariatric surgery: retrospective evaluation and literature review. Rev Bras Anestesiol 2006; 56: 205-22.

6. Altermatt FR, Muñoz HR, Delfino AE, Cortínez LI. Pre-oxygenation in the obese patient: effects of position on tolerance to apnoea. Br J Anaesth 2005; 95: 706-9.

7. Shah A, Uribe J, Katz PO. Gastroesophageal reflux disease and obesity. Gastroenterol Clin North Am 2005; 34: 35-43.

8. Ogunnaike BO, Jones SB, Jones DB, Provost D, Whitten CW. Anesthetic considerations for bariatric surgey. Anesth Analg 2002; 95: 1793-805.

9. Nguyen NT, Wolfe BM. The physiologic effects of pneumoperitoneum in the morbidly obese. Ann Surg 2005; 241: 219-26.

10. McNatt SS, Longhi JJ, Goldman CD, McFadden DW. Surgery for obesity: a review of the current state of the art and future directions. J Gastrointest Surg 2007; 11: 377-97.

11. White RH, Keenan CR. Effects of race and ethnicity on the incidence of venous thromboembolism. Thromb Res 2009; 123 Suppl 4: S11-7. 